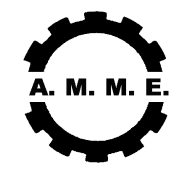

\title{
DYNAMIC PERFORMANCE OF AN ELECTROHYDRAULIC SERVO ACTUATOR WITH CONTACTLESS CONTROLLED SPOOL
}

\author{
M. Metwally ${ }^{*}$, G.Rabie ${ }^{* *}$, N. Girgis ${ }^{* * *}$ andl. Saleh ${ }^{* * *}$
}

\begin{abstract}
This paper deals with the analysis of dynamic behavior of an electrohydraulic proportional actuator. The studied system consists of an electromagnetic proportional solenoid, a hydraulic cylinder and a directional control valve incorporating a nonconventional hydraulic amplifier. The spool of the directional control valve is driven by the pressure force developed by the built-in hydraulic amplifier. The peculiarity of the spool-solenoid interaction, forces the spool to have the same displacement as that of the solenoid core. The solenoid force is only needed to activate the hydraulic amplifies. This leads to the use of a solenoid of small dimensions. The system has been studied theoretically by deducing a nonlinear mathematical model, and developing a computer simulation program.
\end{abstract}

The used solenoid is of the stroke controlled type. The steady state currentdisplacement relation has been measured and considered in the simulation program. The transient response of the solenoid has been measured experimentally and used to validate the simulation program. The proportional actuator has been studied in a closed loop control system. A PID controller has been designed to improve the closed loop dynamics. The performance of the system has been studied theoretically on the base of the developed simulation program.

\section{KEY WORDS}

Electrohydraulic, Proportional, Electromagnetic, Solenoid, Valve, Actuator, Spool, PID, Simulation, Closed Loop.

\footnotetext{
${ }^{*}$ Ph. D. Applicant, Egyptian Armed Force.

${ }^{* *}$ Professor, Modern Academy for Engineering and Technology

*** Professor, Military Technical Collage.

**** Professor, Military Technical Collage.
} 


\section{NOMENCLATURE}

A Cross-sectional area of the core; $m 2$

$\mathrm{A}(\mathrm{x}) \quad$ Valve outlet area, $\mathrm{m} 2$

AA Piston area at cab side, $\mathrm{m} 2$

$\mathrm{AB} \quad$ Piston area at rod side, $\mathrm{m} 2$

Aco Solenoid core cross section area, $\mathrm{m} 2$

Arc Spool radial clearance area, $\mathrm{m} 2$

As Spool cross-sectional area, $\mathrm{m} 2$

Ath Throttle area, $\mathrm{m} 2$

B Bulk modulus of oil, $\mathrm{Pa}$

C Pull; (N/m2)/(A-turn/m)

C Spool radial clearance, $m$

Ds Spool diameter, $\mathrm{m}$

$\mathrm{f} \quad$ Friction coefficient; $\mathrm{kg} / \mathrm{s}$

F Solenoid spring loading force; $N$

FAseat Actuator seat reaction force, $\mathrm{N}$

fAseat Actuator seat friction coefficient, $\mathrm{Ns} / \mathrm{m}$

FL Load force, N

FM Magnetic pull force acting on the solenoid core; $\mathrm{N}$

FP Pressure force acting on the solenoid core due to valve pressure ; $N$

fp Piston friction coefficient, $\mathrm{Ns} / \mathrm{m}$

fseat Seat friction coefficient; $\mathrm{Ns} / \mathrm{m}$

Fseat Reaction seat force on the solenoid core due to valve spool; $\mathrm{N}$

i Supplied current; $A$

ib Feed back current, $A$

ie $\quad$ Error current signal, A

K Stiffness of the core loading spring; $\mathrm{N} / \mathrm{m}$

KAseat Equivalent actuator seat stiffness, $\mathrm{N} / \mathrm{m}$

$\mathrm{kb} \quad$ Feed back gain, $\mathrm{A} / \mathrm{m}$

Kseat Equivalent seat stiffness; $\mathrm{N} / \mathrm{m}$

I Length of the solenoid; $m$

mc Solenoid core mass; $\mathrm{kg}$

$\mathrm{mp} \quad$ Piston mass, $\mathrm{kg}$

$\mathrm{N} \quad$ Number of turns of coil

$\mathrm{P} 1 \quad$ Supply Pressure, $\mathrm{Pa}$

PA\&PB Hydraulic cylinder pressures, $\mathrm{Pa}$

Po Valve return pressure, $\mathrm{Pa}$

$\mathrm{Pv} \quad$ Pressure inside the valve chamber, $\mathrm{Pa}$

$\mathrm{Px} \quad$ Pressure upstream throttle, $\mathrm{Pa}$

Q1 Flow rate in the inlet orifice, $\mathrm{m} 3 / \mathrm{s}$

Q2 Flow rate in the outlet orifice, $\mathrm{m} 3 / \mathrm{s}$

$\mathrm{Ri} \quad$ Resistance to internal leakage, Ns/m3

VA volume of oil filling the cylinder at cab side, $\mathrm{m} 3$

VB volume of oil filling the cylinder at rod side, $\mathrm{m} 3$

Vo Volume of oil in the spool side chamber, m3

xc Solenoid core displacement ; $m$

xs Sliding hydraulic amplifier displacement ; m 


\section{INTRODUCTION}

The combination between electronics and hydraulic power system leads to better system controllability. The electrohydraulic proportional control system has the advantages of the maximum power to weight ratio and high stiffness of the hydraulic actuators. It has also the advantages of the electronic control systems, specially the high controllability and precision.

Proportional control valves are used as final control elements in the open and closed control system. Their static and dynamic properties have a decisive influence on the overall characteristics of the electrohydraulic control systems. The task of a proportional control valve is to convert the electric input control signal into a proportional hydraulic output signal, i.e. into a pressure or a flow rate. The valves are classified according to their hydraulic output quantities or functions, e.g. proportional pressure valve, proportional throttle valve, and proportional directional control valve.

Proportional solenoids convert electrical energy to mechanical energy through a magnetic field. The magnetic field has a highly nonlinear characteristic and it is difficult to be modeled. There is few literature on the modeling and simulation of proportional solenoids. This is because the early usage of the solenoid was only as ON/OFF control. An example of the usage of the proportional solenoid is the work of Arno Schmitt et al (1989) [1]. He gave an introduction to the practical applications of the stroke-controlled and force-controlled solenoids. N.C. Chueng et al (1996) [2], developed a project aiming to convert a low cost, highly nonlinear switching solenoid into a proportional actuator through an intelligent control. Also, Greg Schoenau et al (1999) [3], used the ordinary least squares and the maximum likelihood parameter estimation techniques to estimate the spring constant and spring pre-compression in the main spool of a solenoid of a proportional hydraulic valve. A.C. Lua et al (2001) [5], presented a study for a new proportional assist ventilation method using a proportional solenoid valve to control air supply to patients suffering from respiratory disabilities.

In the conventional usages of the proportional solenoid, the valve spool is directly driven by the solenoid core. The solenoid should drive the spool against the friction force, spring force, flow force, and inertia force. Generally, large spools require great solenoid force and consequently greater dimensions of solenoid winding. It is seldom to find a direct-acting proportional solenoid works with a proportional valve with spool diameter larger than $9 \mathrm{~mm}$ [4].

\section{DESCRIPTION OF THE EHSA}

This paper describes the new features of a proportional solenoid working in conjunction with proportional valve. The solenoid is of stroke controlled type. It controls the spool through a contactless arrangement. There is no direct contact between the solenoid core and valve spool. An integrated hydraulic amplifier produces the pressure force needed to drive the spool. It is controlled by the displacement of the solenoid core, in a manner analogous the single jet flapper valve. Actually, the spool and core move as one unit. The contactless electrohydraulic proportional valve has the following advantages:

- Usage a relatively small and simple construction proportional solenoid to drive a 
proportional valve spool of large diameter.

- By using a feedback control system, it will give considerable precisions of electrohydraulic servo actuator movement.

- The proportional valves spools are almost entirely machine produced, while the servovalves spools require a great deal of manual lapping and finishing.

- More reliable than the electro servo valve, in a heavy duty conditions, while the clearance and tolerance of servovalves are much tighter than proportional valves.

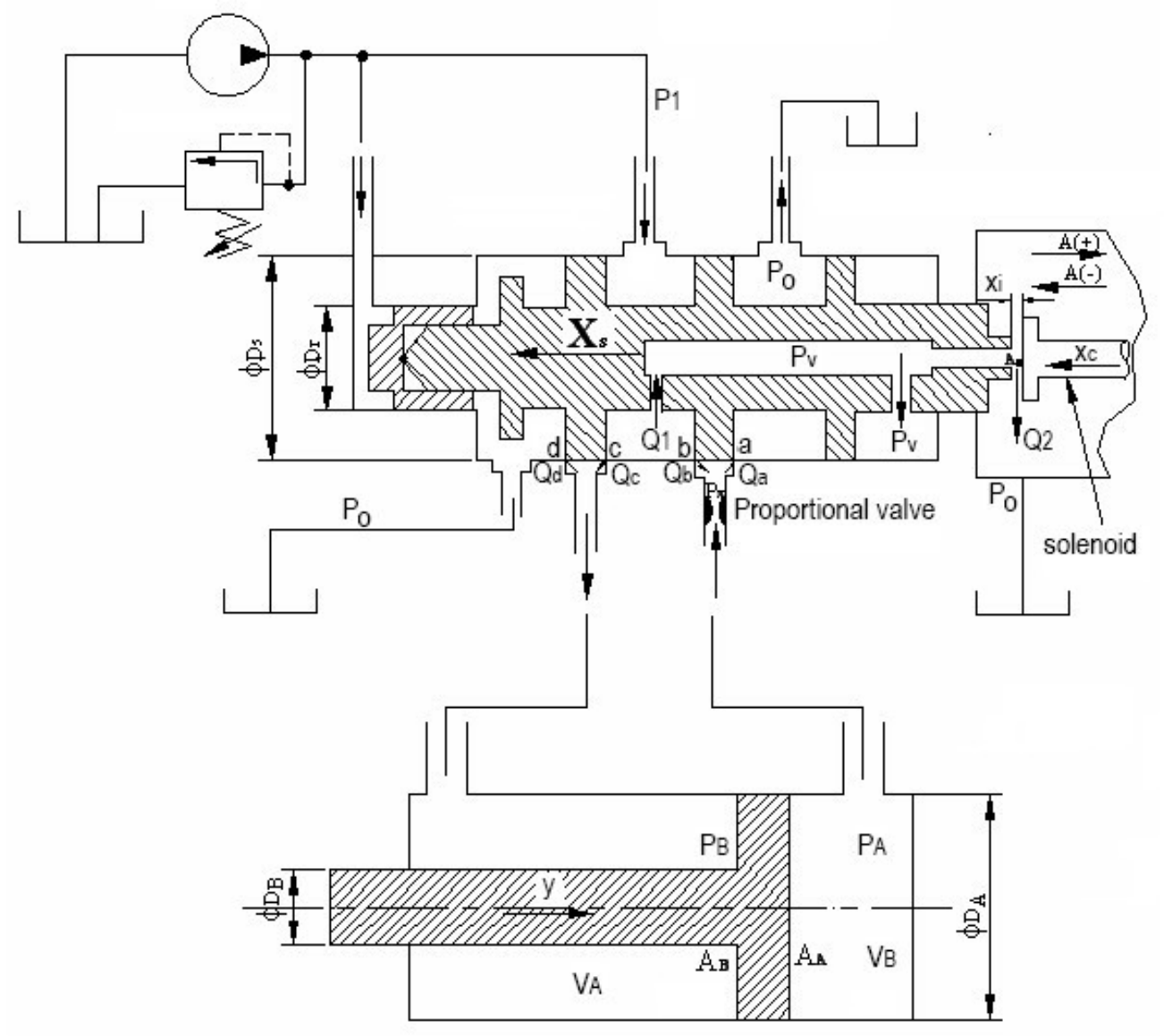

Fig. 1 Functional scheme of the studied electrohydraulic servo actuator.

Figure 1 shows a functional scheme of the studied electrohydraulic servo actuator. It consists of a stroke controlled proportional solenoid, a zero lapping directional control valve and a single rod hydraulic cylinder. The spool of the directional control valve incorporates a hydraulic amplifier which is controlled by the width of the gap separating the amplifier input nozzle and the solenoid core. The hydraulic amplifier acts as a pressure reducer with two restrictors; a fixed nozzle and a variable one. The variable nozzle is controlled by the width of the gap between the spool and solenoid core. The left side of the spool is subjected to the constant supply pressure $\mathrm{p} 1$, while the greater area of the right side is subjected to the reduced pressure pv. At certain gap width, the pressure forces are equal and the spool is at rest. If the core is moved to the left, the gap width decreases, increasing the reduced pressure pv. The increased pressure force acts on the spool and displaces it to the left. The spool settles when the original gap width is reached. The motion of the core to the right increases the gap width, reducing the pressure pv. The spool moves to the right until 
the original gap width is reached. Thus, in the steady state, the gap width has practically a fixed value.

The hydraulic cylinder is controlled by the directional control valve. An additional orifice is added in the line connecting the directional control valve to the piston side to insure the required damping. The piston rod displacement is picked-up and fed back to the electronic controller where it is compared with the input signal. The difference, the error signal, is applied to the solenoid (the feedback arrangement is not illustrated by Fig.1). The piston motion ceases when the error signal becomes null.

\section{THE ELECTROMAGNETIC PROPORTIONAL SOLENOID}

\subsection{Construction and Operation}

The electrical solenoid is a winding of insolated conductor around a movable iron core. When DC current passes through solenoid winding, the iron rod is magnetized. The mutual action of the solenoid field on the magnetic poke created on the core causes the core to move inside the solenoid opposing the force of the internal spring. In the steady state, the core displacement is determined by the equality of the solenoid [FM] and spring forces [F].

$$
\begin{aligned}
& \mathrm{F}_{\mathrm{M}}=\mathrm{CAni} / \mathrm{I} \\
& \mathrm{F}=\mathrm{K} * \mathrm{X}_{\mathrm{c}}
\end{aligned}
$$

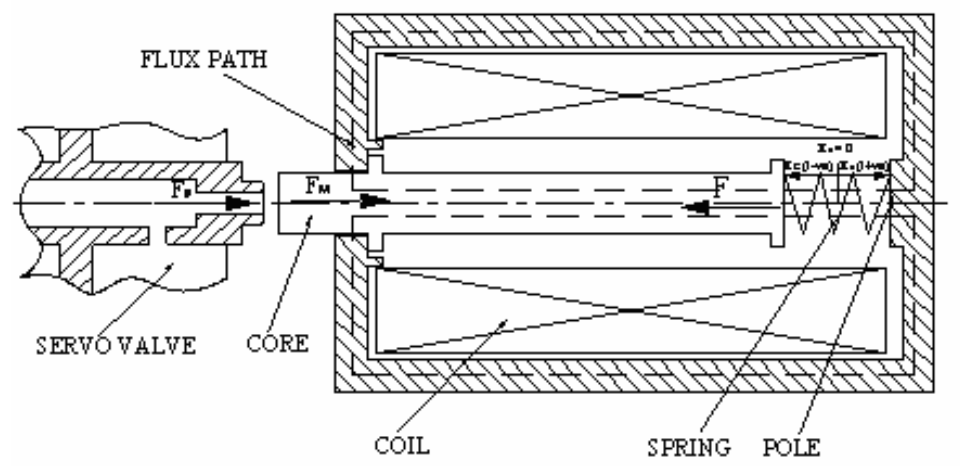

Fig. 2 Proportional solenoid with contact-less connection with the spool

When the energizing current is switched OFF; the core moves back within the solenoid to its extreme out position under the action of the spring. The proportional solenoid under consideration is of DC analogue type. The core displacement is proportional to the current passing through the coil winding. There is no contact between its core and the valve spool as shown in Fig. 2.

In the present application, the solenoid is continuously excited by $300 \mathrm{~mA}$. The resulting solenoid force, together with the spring force, causes the core to move to the mid-point of its possible travel. This position is the initial position of the solenoid core. The core can be controlled to move in or out of the solenoid by increasing or 
decreasing the control current respectively. The displacement-current relation has been difficult to be investigated theoretically, so, it has been evaluated experimentally.

\subsection{Measurement of the Steady State Displacement-Current Relation}

The static characteristic of the solenoid is the relation between the current supplied to the solenoid and the resulting core displacement. The measuring scheme of the steady state core displacement-current relation is shown in Fig. 3. A variable DC power supply has been used to control the solenoid current, while the core displacement has been measured by a dial indicator.

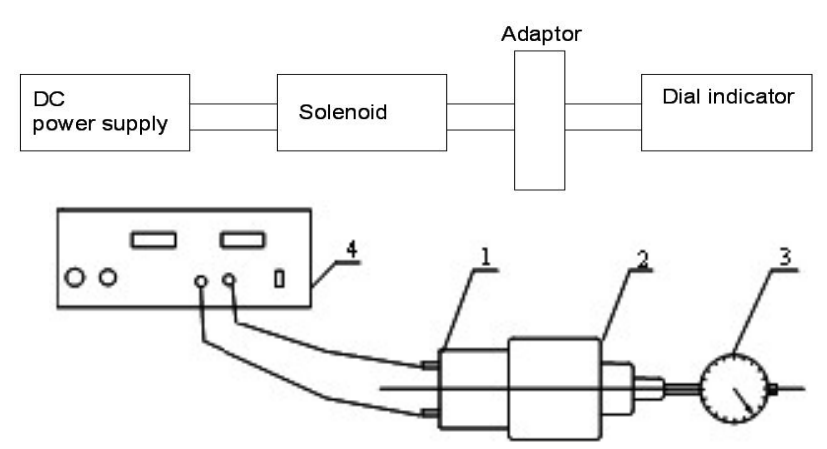

1-Solenoid 2- Adaptor 3- Dial indicator 4- power supply

Fig. 3 Setup of the solenoid static characteristics measurement

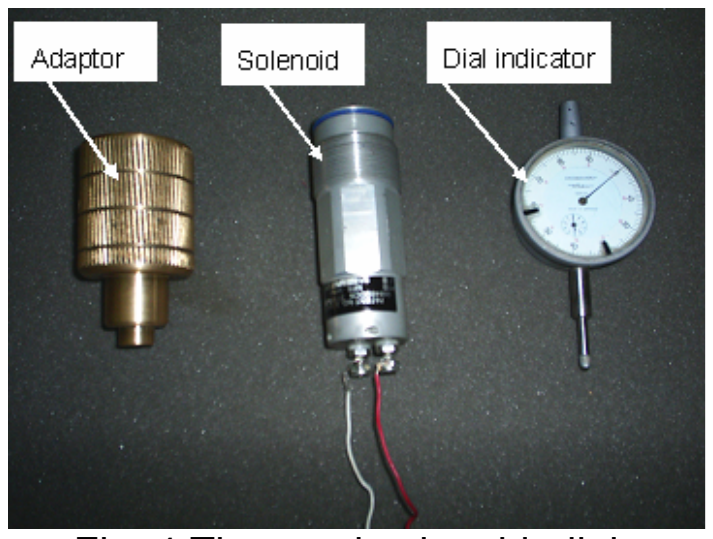

Fig. 4 The used solenoid, dial indicator and adaptor.

A mechanical adaptor is designed to match the solenoid connection to the dial indicator such that the displacement of the solenoid core is directly transferred to the sensing axis of the dial indicator, Fig. 4.

The supplied current to solenoid has been changed in steps of $100 \mathrm{~mA}$ and the resulting core displacement was measured. The results are shown in Fig. 5, which shows effect of magnetic hysteresis in addition to an almost linear force-current relation. 


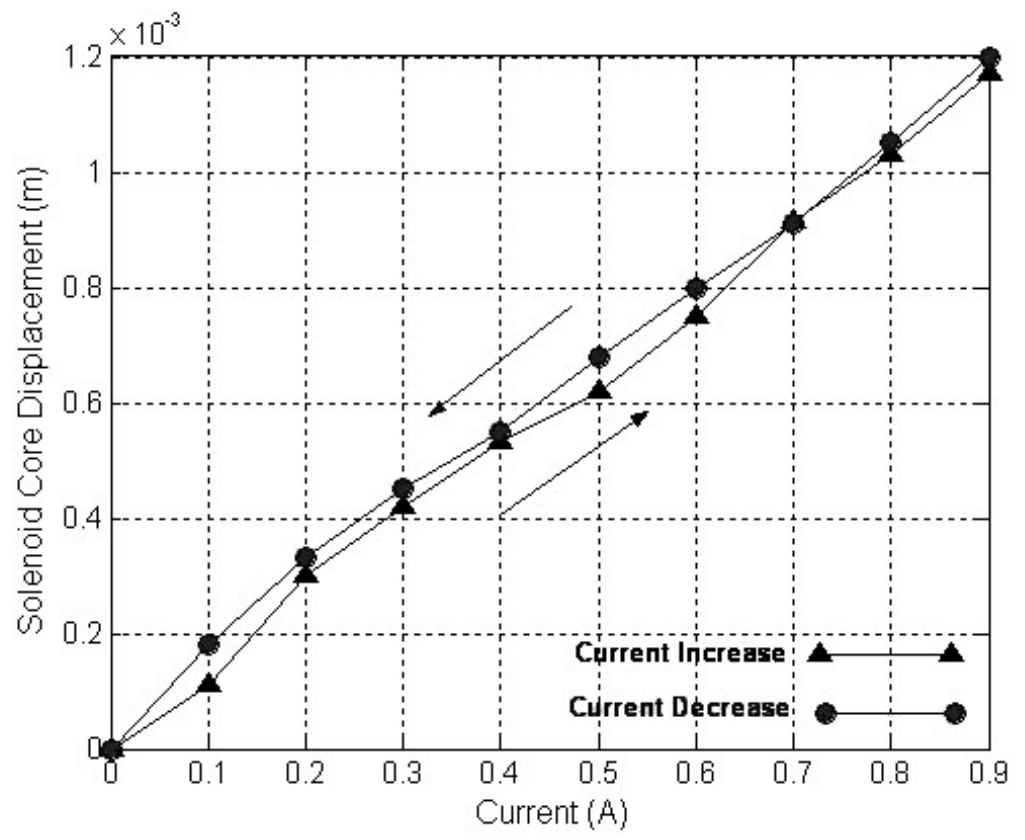

Fig. 5 Proportional solenoid static characteristic due to supplied current

\subsection{Measurement of the Solenoid Transient Response}

The solenoid under consideration has been treated as a black-box system because its internal structure is unknown. The dynamic characteristics of such a system could be obtained by practical testing of the system itself. This is obtained by measuring the solenoid response to a step current excitation.

The experimental set up consists of a DC power supply, a square pulse generator, a linear variable differential transformer (LVDT), and an oscilloscope. An adaptor has been used to couple mechanically the solenoid core with the LVDT. In this case the output signal from the LVDT is directly proportional to the solenoid core displacement. This output electric signal has been indicated on the oscilloscope.

The square pulse generator has been used to switch the solenoid current, in repeated way, between the datum value $(300 \mathrm{~mA})$ and zero value $(0 \mathrm{~mA})$ or between the datum value and maximum value $(700 \mathrm{~mA})$. This will lead to the motion of the core between the initial steady state-point (the mid-point of its travel) and the extreme outer position or between the initial point and the selected extreme inner position respectively. 


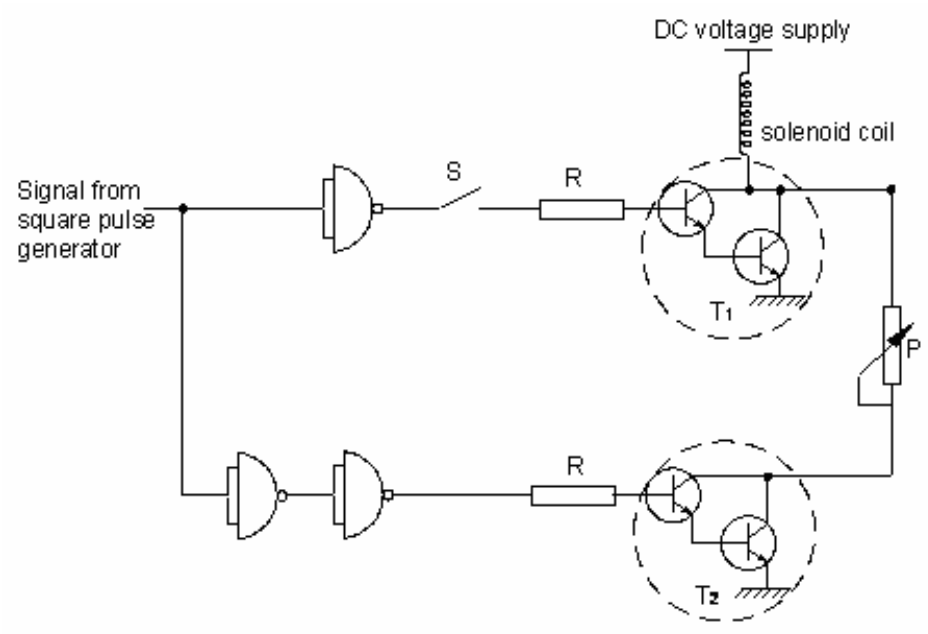

Fig.6. Electronic circuit schematic

The electronic circuit shown in Fig. 6 has been used to control the solenoid current. It consists of two transistor switches T1\&T2 and three logic gates. Each of the transistor switches T1\&T2 is based on two Darlington connected transistors. One of the solenoid terminals is connected to the DC power supply while the 2 nd is earthed through one of the transistor switches T1 or T2. The output of the DC power supply has been adjusted such that when T1 is switched ON, $700 \mathrm{~mA}$ current will pass through the solenoid. The potentiometer $P$ has been adjusted such that when T2 is ON a 300mA will pass through the solenoid and the series potentiometer $P$. The output of the square pulse generator is fed, through the logic gates, out of phase, to T1\&T2 i.e. the input signal to T1 is the complement of that of T2. When switch $s$ is disconnected, theT1 is always noncoducting and T2 switches the solenoid current between $0 \& 300 \mathrm{~mA}$. When switch s is connected, T1\&T2 are connected alternatively. When T1 is ON, $700 \mathrm{~mA}$ flows through solenoid and when T2 is ON 300mA flows.

The LVDT is coupled mechanically to the solenoid core by the adaptor. Its output signal has amplitude indicating the magnitude of the displacement, and phase indicating the direction. A 300mA datum current has been given to the solenoid to let its core to be in the mid way. Any positive or negative current steps supplied to the solenoid is compiling to an electrical signal by LVDT and indicated on the oscilloscope as shown in Fig. 7.

The LVDT output signal has been indicated on the oscilloscope. The transient response of solenoid to step input current of two different magnitudes was measured. The experimental results are plotted in Fig.8. Figure 8a shows the transient response to step up current from 300 to $700 \mathrm{~mA}$, where the core is pulled inside the solenoid. Figure $8 \mathrm{~b}$ shows the transient response to step down current from 300 to $0 \mathrm{~mA}$, where the solenoid core is pushed outside under the action of the spring force. 


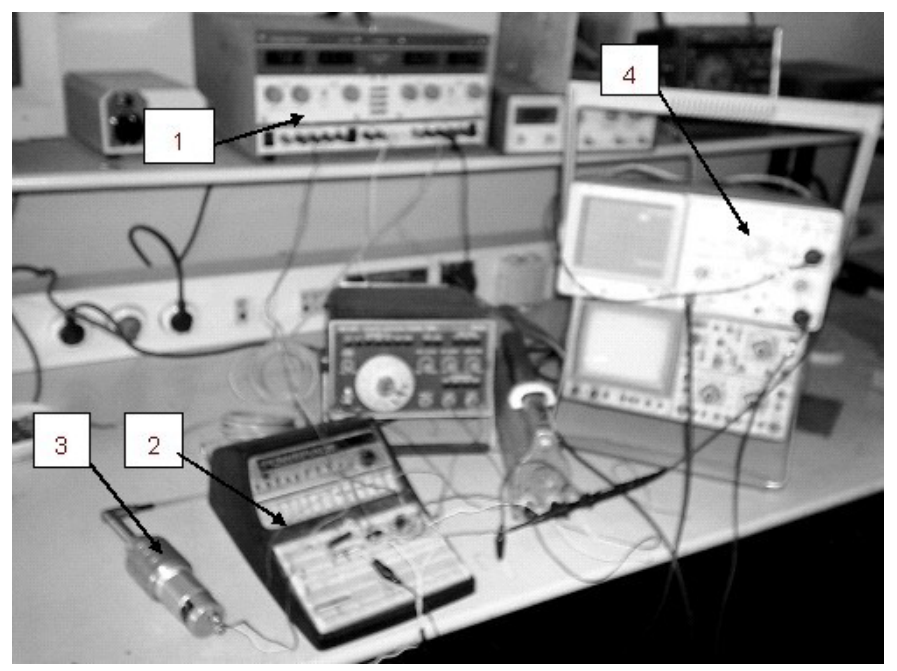

Fig.7 Experimental set-up for the measurement of the transient response of the proportional solenoid

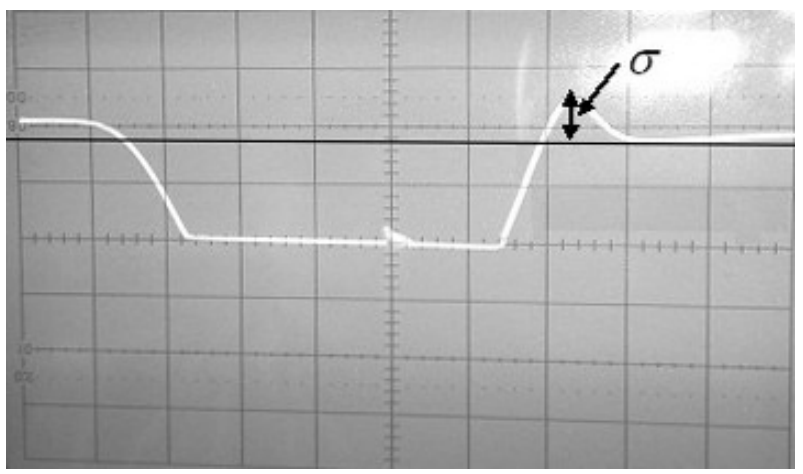

Fig. 8 a. response to step-up current

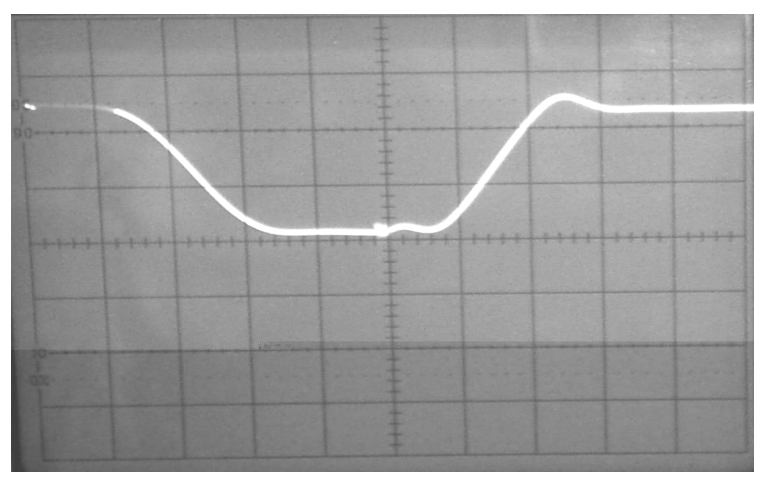

Fig. 8b. Response to step down current

The measured solenoid dynamic response, shown in Fig. 8, is apparently of an under damped second order element. The experimental results were used to identify the solenoid and estimate its parameters. The damped natural frequency $(\omega d)$, period $(\tau)$, and maximum overshoot ratio $(\sigma)$ were determined from the experimental results. The second order transfer function is fully determined by calculating the natural frequency $(\mathrm{n})$, the damping coefficient $(\mathrm{l})$ and the gain $\mathrm{k}$. These parameters were calculated by the following expressions:

$$
\begin{aligned}
& \omega_{\mathrm{d}}=2 \pi / \tau \\
& \zeta=\sqrt{\frac{\{\ln (\sigma)\}^{2}}{\{\ln (\sigma)\}^{2}+\pi^{2}}} \\
& \omega_{\mathrm{n}}=\omega_{\mathrm{d}} / \sqrt{1-\zeta^{2}}
\end{aligned}
$$

The solenoid unknown parameters are, the solenoid core mass [ $\mathrm{m}_{\mathrm{c}}$ ], the solenoid spring stiffness $\left[K_{c}\right]$, and the solenoid friction coefficient $\left[\mathrm{f}_{\mathrm{c}}\right]$. These parameters are related to $\omega_{n} \& \zeta$ of the system through the following equations. 


$$
\omega_{\mathrm{n}}=\sqrt{\frac{\mathrm{K}_{\mathrm{c}}}{\mathrm{m}_{\mathrm{c}}}} \quad \& \quad \zeta=\frac{\mathrm{f}_{\mathrm{c}}}{2 \sqrt{\mathrm{K}_{\mathrm{c}} \mathrm{m}_{\mathrm{c}}}}
$$

There are three unknowns in two equation. Their values have been obtained by trail and error. Within the expected range of the solenoid core mass $\left[\mathrm{m}_{\mathrm{c}}\right.$ ], a number of values are assumed. For each value the corresponding values of $f_{c}, k_{c}$ have been obtained using equation (6). On the base of these values, the transfer function coefficients were determined and used to calculate the step response. The results are plotted with the experimental results in Fig.9. The best agreement between simulation and experimental results was found for the following numerical values.

$$
m_{c}=0.02 \mathrm{~kg}, \mathrm{k}_{\mathrm{c}}=37500 \mathrm{kn} \text {, and } \mathrm{f}_{\mathrm{c}}=30 \mathrm{Ns} / \mathrm{m} \text {. }
$$

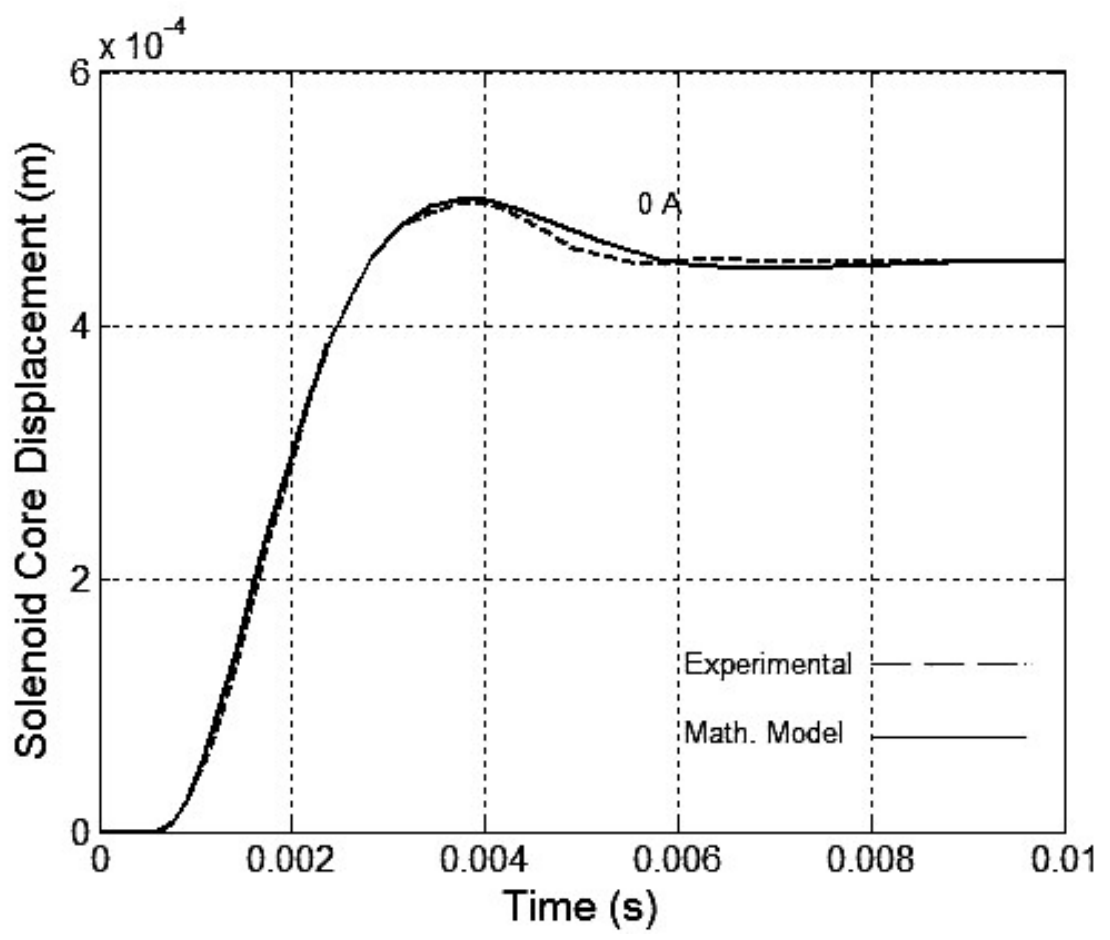

Fig.9 Experimental and theoretical results of transient response of solenoid core displacement to step variation of current, from $0.300 \quad A$

\subsection{Modelingand Simulation of the Proportional Solenoid}

The main function of electromagnetic proportional solenoid is to convert the electric input signal into a proportional mechanical displacement. Neglecting the effect of magnetic hysteresis, the transient response of the solenoid is described by the followingequations:

$$
F_{P}=P_{v} A_{c}
$$




$$
F=m_{c} X_{c}^{*}+f X_{c}+K X_{c}+F_{P}+F_{\text {seat }}
$$

Where the seat reaction force is given by the following equation:

$$
F_{\text {seat }}= \begin{cases}0 & \text { For }\left|x_{s}-x_{c}\right|>0 \\ K_{\text {seat }}\left|x_{s}-x_{c}\right|-f_{\text {seat }}\left(\dot{x}_{c}+\dot{x}_{s}\right) & \text { For }\left|x_{s}-x_{c}\right| \leq 0\end{cases}
$$

Using the SIMULINK package, a simulation program has been developed, based on equations 7 to 9 .

\section{PROPORTIONAL DIRECTIONAL CONTROL VALVE (EHPV)}

\subsection{Mathematical Model of the EHPV}

Neglecting the local losses through transmission line, the electrohydraulic proportional directional control valve could be described by the following equations.

The following equations give the Flow rate though the restrictions of the hydraulic amplifier.

$$
\begin{aligned}
& Q_{1}=C_{d} A_{1} \sqrt{\frac{2}{\rho}\left(P_{1}-P_{v}\right)} \\
& Q_{2}=C_{d} A_{2} \sqrt{\frac{2}{\rho}\left(P_{v}-P_{0}\right)}
\end{aligned}
$$

The application of the continuity equation to the hydraulic amplifier chambers results in the following relation.

$$
Q_{1}-Q_{2}+A_{s} \frac{d x_{s}}{d t}-\frac{V_{0}-A_{s} x_{s}}{B} \frac{d P_{v}}{d t}=0
$$

The motion of the two stages proportional directional control valve spool could be described by the following equation.

$P_{1} A_{r}-P_{v} A_{s}-P_{v} A_{n}=m_{s} \frac{d^{2} x_{s}}{d t^{2}}+f_{s} \frac{d x_{s}}{d t}+F_{j}+F_{\text {seat }}$

Where the jet reaction (Bernoully) force is given by: 


$$
F_{j}= \begin{cases}\frac{\rho Q_{b}^{2}}{C_{c} A_{b}}+\frac{\rho Q_{d}^{2}}{C_{c} A_{d}} & \text { For } x>0 \\ \frac{\rho Q_{a}^{2}}{c_{c} A_{a}}+\frac{\rho Q_{c}^{2}}{c_{c} A_{c}} & \text { For } x<0\end{cases}
$$

The deduced mathematical relations were used to develop a simulation program for the studied valve. the simulation program has been used to calculate the step response of the studied valve.

\subsection{Transient Response of the EHPV}

When the solenoid is energized by a supply current of $300 \mathrm{~mA}$, the pressure $\left(P_{\mathrm{v}}\right)$ generated from pressure amplifier controls the proportional valve spool to be in its null (equilibrium) position. When the solenoid is energized by any different current, a changing in pressure will appear. Due to change in pressure $\left(P_{v}\right)$, the force acting on the valve spool will vary, causing valve spool to move from its null position. It will not stop until this force returns to its equilibrium value again leaving the valve spool in a new position.

The transient response of the valve was investigated theoretically by applying step variation of the control current. The input current steps are from 300 to $0 \mathrm{~mA}$ and from 0 to $700 \mathrm{~mA}$, as shown in Fig. 10. The dependence of valve pressure $\left(P_{v}\right)$ on the solenoid current is shown by Fig. 11. The transient response of the EHPV to step variations of solenoid current is shown in Fig. 12. These results show that by the end of the transient period, the spool displacement equals that of the solenoid core. and core displacements. The settling time of the core is within $5 \mathrm{~ms}$, while that of the spool is within $7 \mathrm{~ms}$.

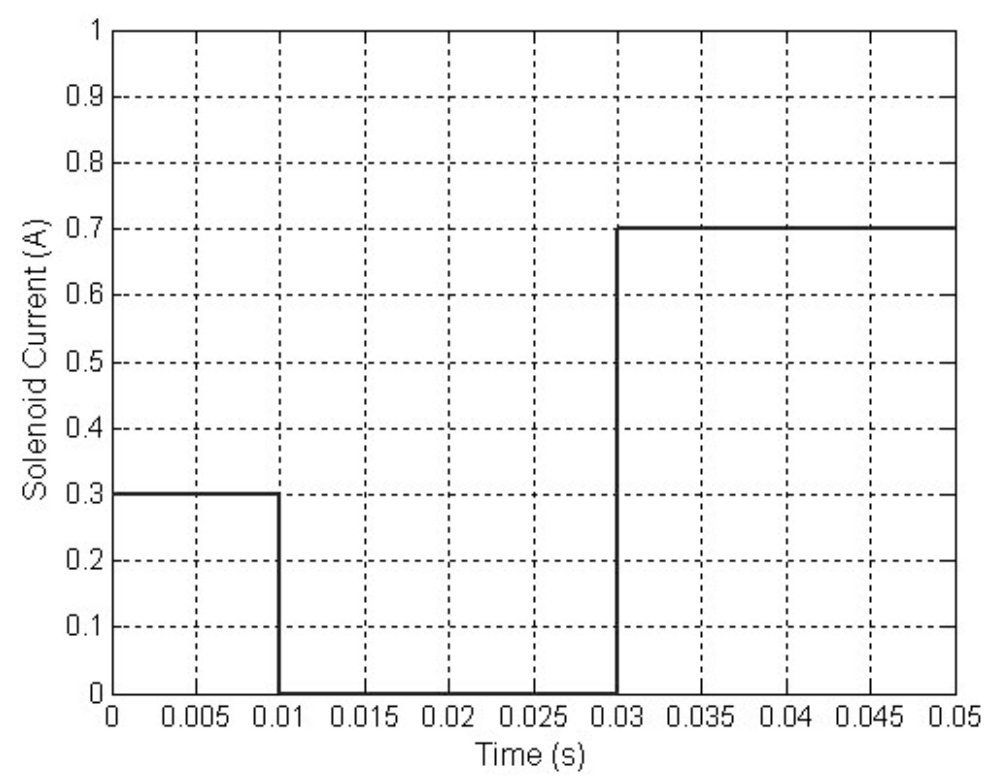

Fig. 10 solenoid current variation with time 


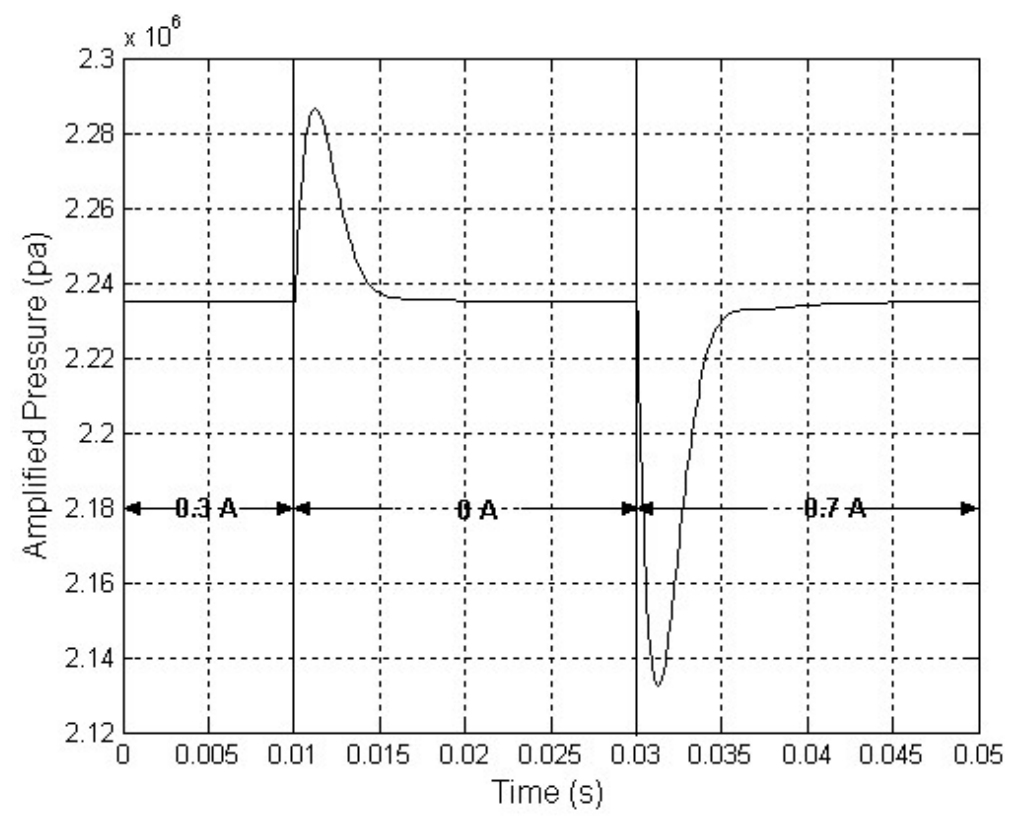

Fig. 11 Pressure generated in EHPV due to solenoid supplied current.

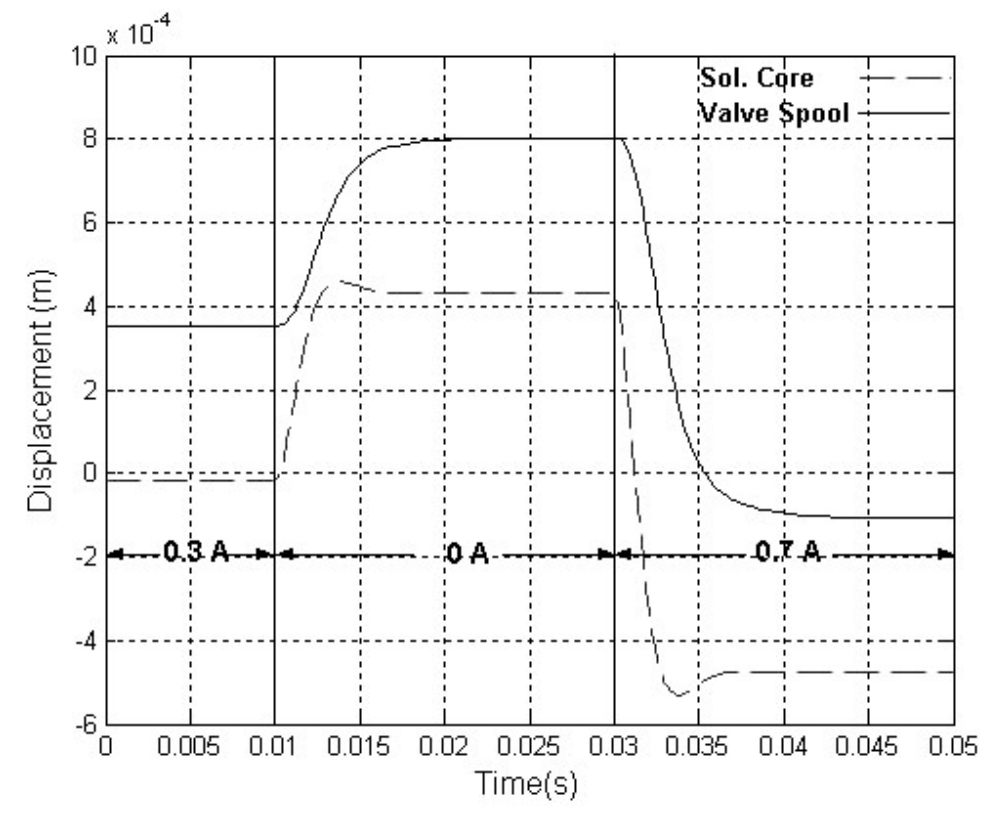

Fig. 12 EHPV dynamic response with the proportional solenoid supplied current

\section{ELECTROHYDRAULIC SERVO ACTUATOR (EHSA)}

\subsection{Construction and Operations}

Figure 1 shows a functional scheme of the studied electrohydraulic servo actuator (EHSA). It consists of an electrohydraulic proportional valve EHPV and a double acting hydraulic cylinder. The system is equipped with a feed back arrangement (Not 
illustrated by Fig.1). The hydraulic cylinder piston displacement is picked up by an LVDT and fed back to the controller

\subsection{Mathematical Model of EHSA:}

In addition to the previously given equations describing the EHPV, the following equations has been deduced to describe the EHSA.

The flow rates through the valve restriction areas.

$$
\begin{aligned}
& Q_{a}=C_{d} A_{a} \sqrt{\frac{2}{\rho}\left(P_{x}-P_{0}\right)} \\
& Q_{b}=C_{d} A_{b} \sqrt{\frac{2}{\rho}\left(P_{1}-P_{x}\right)} \\
& Q_{c}=C_{d} A_{c} \sqrt{\frac{2}{\rho}\left(P_{1}-P_{B}\right)} \\
& Q_{d}=C_{d} A_{d} \sqrt{\frac{2}{\rho}\left(P_{B}-P_{0}\right)} \\
& Q_{\text {th }}=C_{d} A_{\text {th }} \sqrt{\frac{2}{\rho}\left(P_{A}-P_{x}\right)}
\end{aligned}
$$

The restriction areas are given by the following relations:

$$
\begin{aligned}
& \begin{cases}A_{b}=A_{d}=A_{r c}+A(x)=\pi\left(D_{s}-C\right) \sqrt{C^{2}+X^{2}} & \text { For } X_{S}<0 \\
A_{a}=A_{c}=A_{r c}=\pi\left(D_{s}-C\right) \cdot C & \end{cases} \\
& \begin{cases}A_{a}=A_{c}=A_{r c}+A(x)=\pi\left(D_{s}-C\right) \sqrt{C^{2}+X^{2}} & \text { For } X_{S}>0 \\
A_{b}=A_{d}=A_{r c}=\pi\left(D_{s}-C\right) \cdot C & \end{cases}
\end{aligned}
$$

Applying the continuity equation to the cylinder chambers, considering the internal leakage and neglecting the external leakage, the following equations have been obtained:

$$
\begin{aligned}
& Q_{b}-Q_{a}+Q_{A}-\frac{V_{x}}{B} \frac{d P_{x}}{d t}=0 \\
& Q_{c}-Q_{d}-A_{B} \frac{d y}{d t}+\frac{\left(P_{A}-P_{B}\right)}{R_{i}}-\frac{V_{B}+A_{B} y}{B} \frac{d P_{B}}{d t}=0 \\
& A_{A} \frac{d y}{d t}-Q_{A}-\frac{V_{A}+A_{A} y}{B} \frac{d P_{A}}{d t}=0
\end{aligned}
$$


The pressure force drives the piston. The piston moves under the action of pressure, viscous friction, inertia and external forces. Its motion is described by the following equation:

$$
P_{B} A_{B}-P_{A} A_{A}+F_{L}=m_{P} \frac{d^{2} y}{d t^{2}}+f_{P} \frac{d y}{d t}+F_{\text {Aseat }}
$$

The seat reaction force is given by:

$$
F_{\text {Aseat }}= \begin{cases}K_{\text {Aseat }}\left(y-y_{\text {max }}\right)+f_{\text {Aseat }} \frac{d y}{d t} & \text { for } y \geq y_{\text {max }} \\ 0 & \text { for } y_{\text {min }} \prec y \prec y_{\text {max }} \\ K_{\text {Aseat }}\left|y-y_{\text {min }}\right|+f_{\text {Aseat }} \frac{d y}{d t} & \text { for } y \leq y_{\text {min }}\end{cases}
$$

The effective current connected to the solenoid is given by the following:

$$
\mathrm{i}_{\mathrm{e}}=\mathrm{i}-\mathrm{i}_{\mathrm{b}} \text {, where } \mathrm{i}_{\mathrm{b}}=\mathrm{k}_{\mathrm{b}}{ }^{*} \mathrm{y}
$$

\subsection{Simulation Program}

Equations 1 to 25 were used to develop a computer simulation program, using the SIMULINK PROGRAM. The simulation program has been used to calculate the transient response of the EHSA.

The transient response of the EHSA has been calculated by the simulation program for open loop connection. In this case, any change in the solenoid current will lead to a corresponding displacement in the valve spool before the pressure $\mathrm{P}$ returns back to its steady state value. The new spool position leads to a continuous motion of the actuator piston to its extreme position. The piston speed changes with the spool restriction area, which depends on the spool position. Figure 13 shows the simulation results for EHSA transient response for different magnitudes of step current. 


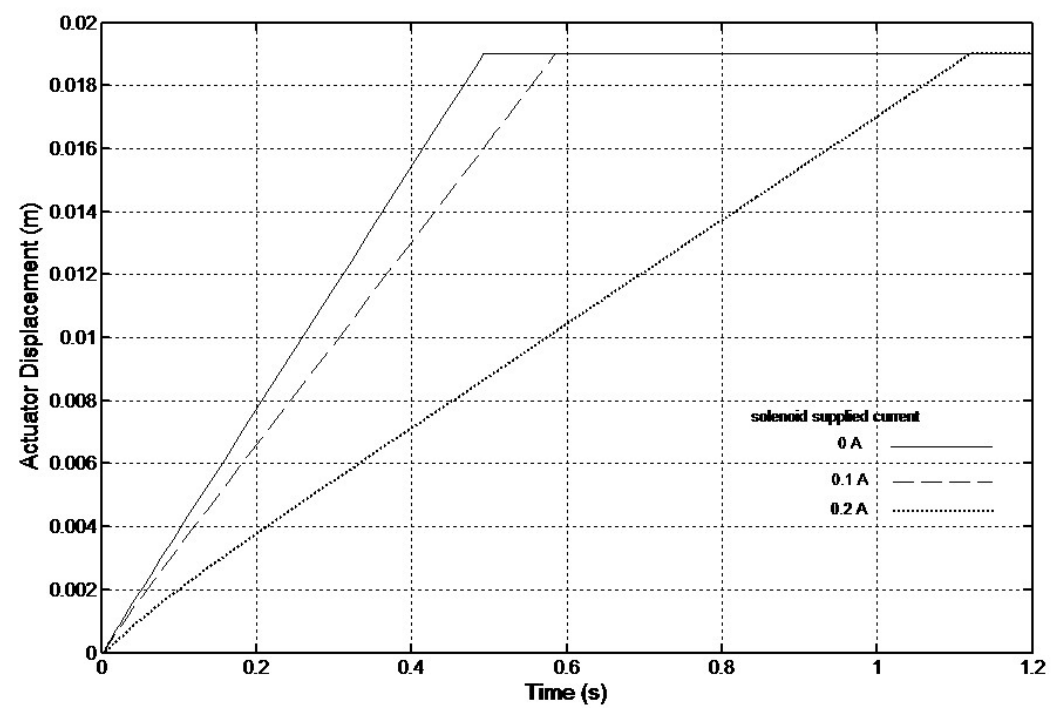

Fig. 13 Step response of EHSA for different steps of applied current.

The closed loop of the studied servoactuator is equipped with a PI controller. The PI controller is described by:

$u(t)=K\left\{e(t)+\frac{1}{T_{i}} \int_{0}^{t} e(\tau) d \tau\right\}$

The controller parameters are the proportional gain $\mathrm{K}$ and the integral time Ti. The numerical values and tuning process of these parameters were carries out according to Ziegler and Nichols. The tuning of PI controller improved radically both of the system stability and precision. The numerical values of the tuned controller were found to be: $\mathrm{K}=1, \mathrm{Ti}=0.0005 \mathrm{~S}$. These tuned parameters produced the almost linear steady state relation ship between the control current and actuator displacement, Fig. 14 . 


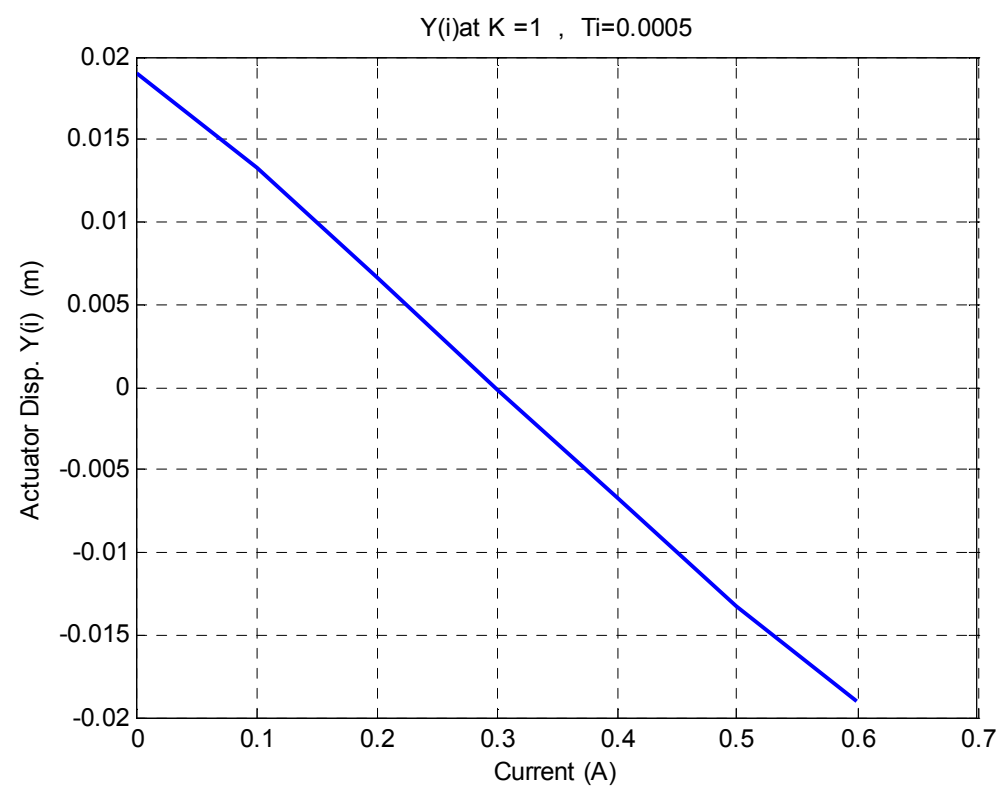

Fig. 14. Steady state relation between the control current and actuator displacement with PI controller

\section{CONCLUSIONS}

This dynamic behavior of an electrohydraulic servoactuator was investigated theoretically and experimentally. The studied valve is controlled by a proportional solenoid, controlling the valve spool through a contactless arrangement. The study included the development of nonlinear mathematical models for the valve elements. The mathematical models were used to develop the computer simulation programs. The transient response of the pilot stage was measured and compared with the simulation results. The good agreement between the simulation and experimental results validated the simulation program of the pilot stage.

The static and dynamic performances of the servoactuator were investigated theoretically using the simulation program.

The study showed that the spool of the pilot stage follows the solenoid core displacement with time delay less than $2 \mathrm{~ms}$. The servoactuator presents almost linear steady state displacement-current relation.

\section{REFERENCES}

[1] Arno Schmitt et. al., Proportional and Servo Valve Technology, Mannesmann Rexroth Gmbh, 1989

[2] N.C. Chueng et. al., "Modeling of a Non Linear Solenoid Towards the Development of a Proportional Actuator", IEEE Industrial Electronic Society annual general, 1996

[3] G. Schoenau, et. al., "Parameter Estimation in a Solenoid Proportional Valve 
Using OLS and MLH Techniques", Mechanical Engineering Department of University of Saskatchewan, Canada,1999

[4] F.D.Norvelle, Electrohydraulic Control System, Prentice Hall inc., New jersey, USA, 2000

[5] A.C. Lua et. al., "Proportional assist ventilation system based on proportional solenoid valve control", transaction of ELESVER, Journal of Medical Engineering \& Physics, 2001(23), pp381-389

[6] M.Galal RABIE, Fluid power Engineering, Published by the author, Cairo, 2005.

[7] M.Galal RABIE, Automatic Control for Mechanical Engineering Students, Published by the author, Cairo, 2005. 